\title{
The time course of contextual influences during lexical ambiguity resolution: Evidence from distributional analyses of fixation durations
}

\author{
Heather Sheridan • Eyal M. Reingold
}

Published online: 11 May 2012

(C) Psychonomic Society, Inc. 2012

\begin{abstract}
In the lexical ambiguity literature, it is wellestablished that readers experience processing difficulties when they encounter biased homographs in a subordinateinstantiating prior context (i.e., the subordinate bias effect). To investigate the time course of this effect, the present study examined distributional analyses of first-fixation durations on 60 biased homographs that were each read twice: once in a subordinate-instantiating context and once in a dominant-instantiating context. Ex-Gaussian fitting revealed that the subordinate context distribution was shifted to the right of the dominant context distribution, with no significant contextual differences in the degree of skew. In addition, a survival analysis technique showed a significant influence of the subordinate versus dominant contextual manipulation as early as $139 \mathrm{~ms}$ from the start of fixation. These results indicate that the contextual manipulation had a fast-acting influence on the majority of fixation durations, which is consistent with the reordered access model's assumption that prior context can affect the lexical access stage of reading.
\end{abstract}

Keywords Reading $\cdot$ Eye movements $\cdot$ Distributional analysis $\cdot$ Lexical ambiguity

Readers frequently encounter homographs (e.g., bank), which have multiple meanings associated with a single orthographic form. Competing models of lexical ambiguity resolution agree that readers use contextual information to determine the relevant meaning of a homograph (e.g., the

\section{H. Sheridan $(\triangle) \cdot$ E. M. Reingold}

Department of Psychology, University of Toronto at Mississauga,

3359 Mississauga Road N. RM 2037B,

Mississauga, Ontario, Canada, L5L 1C6

e-mail: heather.sheridan@utoronto.ca money vs. the river meaning of bank), but controversy has surrounded the temporal locus of contextual influences. On one side of the debate, the modular or autonomous models (e.g., Fodor, 1983; Forster, 1979) have argued that the preceding context does not influence lexical access. Specifically, all meanings of an ambiguous word are accessed exhaustively, regardless of context, and meaning selection is accomplished at the postlexical integration stage. On the other side of the debate, interactive models (e.g., McClelland \& Rumelhart, 1981; Morton, 1969) have argued that context can constrain lexical access such that only the contextually relevant meaning is accessed (i.e., selective access).

Historically, the interactive and modular models were tested empirically using the cross-modal priming task (e.g., Swinney, 1979). In this task, subjects listened to a disambiguating context and an ambiguous word, while simultaneously responding to a visual prime word that was related to either the contextually relevant or irrelevant meaning of the ambiguous word. The ambiguous word primed both meanings if the prime was presented immediately after the ambiguous word. However, if the prime was delayed by $200 \mathrm{~ms}$, only the contextually relevant meaning showed priming. This pattern of results was largely interpreted as supporting the modular view that contextual influences are postlexical (Fodor, 1983; Forster, 1979; but see Lucas, 1999).

However, the introduction of eye tracking to the study of lexical ambiguity resolution by Rayner and Duffy (1986) has led to additional findings that cannot be easily explained by either the modular or the interactive models. A comprehensive review of the findings from eye movement studies of lexical ambiguity is beyond the scope of the present article (for a review, see Duffy, Kambe \& Rayner 2001). Briefly, eye-tracking studies have manipulated aspects of both the context and the homographs. A key aspect of the homographs is the relative frequency of the various 
meanings (i.e., meaning dominance; Hogaboam \& Perfetti, 1975). Specifically, eye-tracking studies have contrasted balanced homographs, which have two approximately equally common meanings, with biased homographs, which have one highly dominant meaning and one or more subordinate meanings. In addition, the preceding context was constructed either to be neutral (i.e., no disambiguating information precedes the homograph) or to instantiate one (or more) of the homograph's meanings. In most lexical ambiguity studies, fixation times on homographs were compared with fixation times on unambiguous control words that were embedded in the same sentence frames (e.g., Duffy, Morris \& Rayner 1988), although several studies have instead used homographs as their own controls by examining fixation times for the same homographs across different context conditions (Dopkins, Morris, \& Rayner, 1992; Rayner, Cook, Juhasz, \& Frazier, 2006; Rayner \& Frazier, 1989; Sheridan, Reingold, \& Daneman, 2009).

Importantly, certain combinations of the meaning dominance and context variables produced longer fixation times on the homographs, relative to control words (i.e., ambiguity-related processing delays), even when the homographs and their controls were equated on relevant variables such as word frequency and word length. For example, when the preceding context instantiated the subordinate meaning, fixation times were longer on biased homographs than on control words (Duffy et al., 1988). This effect was later termed the subordinate bias effect (SBE) by Pacht and Rayner, (1993) and has been the focus of extensive empirical and theoretical efforts in the literature (Binder, 2003; Binder \& Rayner, 1999; Dopkins et al., 1992; Duffy et al., 2001; Duffy et al., 1988; Folk \& Morris, 2003; Kambe, Rayner, \& Duffy, 2001; Pacht \& Rayner, 1993; Rayner, Binder, \& Duffy, 1999; Rayner et al., 2006; Rayner \& Frazier, 1989; Rayner, Pacht, \& Duffy, 1994; Sereno, 1995; Sereno, O’Donnell, \& Rayner, 2006; Sereno, Pacht, \& Rayner, 1992; Wiley \& Rayner, 2000). In addition to the $\mathrm{SBE}$, there are also processing delays relative to controls whenever balanced homographs are placed in a neutral context. However, no delays occur if balanced homographs are placed in a disambiguating context or if biased homographs are placed in either a neutral or dominantinstantiating context. Both strong interactive accounts and strong modular accounts cannot explain this pattern of empirical findings. Interactive models are inconsistent with the subordinate bias effect, because a preceding disambiguating context should result in selective access of the subordinate meaning without any processing delays. Modular accounts cannot explain the findings that fixation times vary depending on the type of preceding context (i.e., neutral, subordinate instantiating, dominant instantiating).

Consequently, two additional models were proposed to explain the full pattern of eye-tracking results: the reordered access model (Duffy et al., 1988) and the integration model (Rayner \& Frazier, 1989). Specifically, the reordered access model postulates that lexical access is exhaustive, and the order by which meanings are accessed is determined by both preceding contextual information (i.e., meanings instantiated by the preceding context are accessed faster) and meaning dominance (i.e., more common meanings are accessed faster). When these factors or their interaction cause two or more meanings to become available at approximately the same time, competition between the meanings leads to processing delays. For example, when the preceding context supports the subordinate meaning of a biased homograph, it speeds up access to that meaning and causes the subordinate and dominant meanings to become available within the same time window, resulting in the SBE.

In contrast, the integration model (Rayner \& Frazier, 1989) proposes that context does not affect lexical access. Instead, the order by which meanings are accessed is determined exclusively by meaning dominance, and context is postulated to affect a postlexical integration stage by facilitating the integration of contextually relevant meanings. According to the integration model, the SBE occurs because integration of the dominant meaning fails and the processor must wait for the subordinate meaning to become available before integration can proceed.

Although both of the models can explain the bulk of the eye movement findings, including the SBE, the results of several empirical studies (Dopkins et al., 1992; Sereno, 1995; Sereno, Brewer, \& O'Donnell, 2003; Sheridan et al., 2009) and simulations (Duffy et al., 2001; Reichle, Pollatsek, \& Rayner, 2007) have favored the reordered access model. For example, in order to distinguish between the two models, Dopkins et al. placed biased homographs (e.g., speaker) in several different context conditions. In this experiment, the most critical condition for testing the two models was the positive condition, in which the preceding context highlighted semantic features of the subordinate meaning but was still consistent with the dominant meaning (e.g., "Inaudible as a result of the static, the speaker was completely rewired by the technician"). The integration model predicts that readers should initially integrate the dominant meaning, because the positive context does not rule out the dominant meaning. In contrast, the reordered access model predicts that readers should initially integrate the subordinate meaning, because the positive context should "boost" the subordinate meaning such that it can compete with the dominant meaning. The reordered access model's prediction was supported by Dopkins et al. and by a related follow-up study by Sheridan et al. (2009). Specifically, Dopkins et al. contrasted the positive context with a neutral control condition, while Sheridan et al. (2009) contrasted the positive context with a dual-meaning (i.e., pun) control condition that highlighted semantic features of both 
the dominant and subordinate meanings without ruling out either meaning. Relative to these control conditions, the positive context produced longer fixation times on the homograph itself, while simultaneously producing shorter fixation times in a later disambiguating region that clearly instantiated the subordinate meaning. The reordered access model can explain this pattern of results by assuming that readers in the positive condition successfully integrated the subordinate meaning upon encountering the homograph and, thus, did not experience difficulty when they later encountered the disambiguating material.

In addition to the approach used by Dopkins et al. (1992) and Sheridan et al. (2009), another way to distinguish between the models is to test their predictions concerning the time course of contextual influences. Critically, the reordered access model proposes an earlier locus of contextual effects during lexical access then does the integration model, which instead assumes that contextual influences are postlexical. Consistent with the reordered access model, an event-related potentials (ERP) experiment by Sereno et al. (2003) and a simulation by Reichle et al. (2007) have supported an early locus of contextual influences during lexical ambiguity resolution. Furthermore, several lexical ambiguity and eyetracking studies (e.g., Kambe et al., 2001; Rayner et al., 2006; Sheridan et al., 2009) have observed contextual influences on the duration of the very first fixation on the homograph (i.e., first-fixation duration). Such first-fixation findings are consistent with the reordered access model's assumption that context can influence lexical access. This is because the temporal constraints inherent to saccadic programming in reading (Sereno \& Rayner, 2003) necessitate that in order for a variable to affect first-fixation duration, it must exert at least part of its influence very early on during the fixation.

However, while the first-fixation findings above are suggestive of an early locus of contextual influences during lexical access, they are not definitive evidence, because it also is possible that the first-fixation effects were driven by a subset of trials with very long fixation times. Consequently, the present study's goal was to provide more decisive evidence for an early locus of contextual influences during lexical ambiguity resolution. To accomplish this goal, the present study examined distributional analyses of firstfixation times on biased homographs (e.g., "suit") in a subordinate-instantiating prior context (e.g., "The law firm agreed to represent me in the suit that was filed last week") versus a dominant-instantiating prior context (e.g., "I went to the dry cleaners to pick up the suit that I'm wearing to the wedding"). This contextual manipulation was expected to replicate the SBE, by producing longer fixation times in the subordinate, relative to the dominant, context. To provide time course information about this effect, we employed two distributional analysis techniques: ex-Gaussian fitting (e.g., Staub, 2011; Staub, White, Drieghe, Hollway, \& Rayner,
2010; White \& Staub, in press; White, Staub, Drieghe, \& Liversedge, 2011; White, Warren, Staub, \& Reichle, 2011) and a survival analysis technique (Reingold, Reichle, Glaholt, \& Sheridan, 2012). These distributional analysis techniques were selected because they had recently proven useful for investigating the time course of other lexical variables during reading, including word frequency (Reingold et al., 2012; Staub et al., 2010) and predictability or contextual constraints (Staub, 2011). The present study extended these techniques to the lexical ambiguity literature to help to distinguish between lexical ambiguity models and, more generally, to provide further information about the time course of lexical and contextual influences during reading.

The ex-Gaussian distribution (Ratcliff, 1979) has been previously used to model individual subjects' distributions of fixation durations during reading (Staub et al., 2010). Specifically, the ex-Gaussian distribution is the convolution of a normal distribution and an exponential distribution, with two parameters corresponding to the normal component ( $\mu$, the mean, and $\sigma$, the standard deviation) and a single exponential parameter $(\tau)$. The present study used ex-Gaussian fitting in order to assess whether the SBE's impact on mean first-fixation duration stems from a shift in the location of the normal component (i.e., an effect on $\mu$ ) and/or a change in the degree of skew (i.e., an effect on $\tau$ ). If the SBE stems from a change in the $\mu$ parameter, such a finding would be consistent with the reordered access model's assumption of an early locus of contextual influences, because it would indicate that both short and long fixations were impacted by the contextual manipulation (Staub et al., 2010). However, if the SBE effect primarily reflects a $\tau$ effect, such a finding would be more consistent with the integration model's assumption of late-acting contextual influences, because it would indicate that the contextual manipulation mainly influences long fixations.

In addition to analyzing ex-Gaussian distribution parameters as described above, we also examined survival curves for the dominant and subordinate first-fixation duration distributions. Survival curves have been previously used to examine fixation times in the study of visual expertise in medicine (for a review, see Reingold \& Sheridan, 2011; see also Feng, Miller, Shu, \& Zhang, 2001, for another application of survival curves), and the technique has more recently been introduced to study eye movement control in reading (Reingold et al., 2012; see also Sheridan \& Reingold, in press) and scene processing (Glaholt \& Reingold, in press). Specifically, for a given time $t$, the percentage of first fixations with a duration greater than $t$ are referred to as the percent survival at time $t$. Thus, when $t$ equals zero, survival is at one hundred percent but then declines as $t$ increases and approaches zero percent as $t$ approaches the duration of the longest observed first fixation. Reingold et al. (2012) examined the time course of word frequency effects during reading 
by calculating separate survival curves for first-fixation durations on low-frequency (LF) and high-frequency (HF) target words. They then examined the earliest point in time at which the LF and HF survival curves began to significantly diverge (henceforth referred to as the divergence point). Importantly, Reingold et al. (2012) argued that the divergence point provided an estimate of the earliest significant influence of the word frequency variable. On the basis of their survival curve analyses, they concluded that there is a significant influence of word frequency on fixation duration in normal reading as early as $145 \mathrm{~ms}$ from the start of fixation. Following Reingold et al. (2012), the present study calculated the divergence point for the subordinate and dominant curves in order to provide an estimate of the earliest significant influence of contextual influences during lexical ambiguity resolution. If the subordinate and dominant survival curves showed an early divergence point that was comparable to the previous word frequency findings (Reingold et al., 2012), such a pattern of results would be consistent with the reordered access model's predictions concerning an early locus of contextual influences. However, if the present findings showed a later divergence point, such a finding would support the integration model's predictions concerning a postlexical locus of contextual findings. Thus, the present study was designed to provide further time course information about the SBE in order to distinguish between models of lexical ambiguity resolution.

\section{Method}

\section{Subjects}

All 60 undergraduate students at the University of Toronto who participated were native English speakers and were given either course credit or $\$ 10$ (Canadian) per hour. All had normal or corrected-to-normal vision.

\section{Materials}

Sixty sentence pairs were created such that the preceding context instantiated either the dominant meaning (e.g., "The baker used a large bowl to mix together the dough for the bread") or the subordinate meaning (e.g., "The used car salesman asked me to pay him the dough for the car") of a biased homograph (e.g., "dough"). To ensure that these sentence pairs were matched for predictability, in an independent sample, 20 undergraduate students were given the sentence frames up to but not including the homographs, and they were asked to produce the word that seemed most likely to come next in the sentence. The target homographs were produced $16 \%$ of the time for the dominant sentence frames and $14 \%$ of the time for the subordinate sentence frames, but this small numerical difference was not significant $(t<1)$. In addition, to minimize differences in local context across the sentences, at least one word prior to the homograph was the same for both sentences (average = 1.6 words), and at least one word after the homograph was the same (average $=1.8$ words). The selection of the 60 noun-noun biased homographs that were included in the sentence pairs was based on the results of norming data collected from an independent sample of 108 subjects (minimum number of observations per item $=10$ ). The norming procedure involved the subjects' writing the first word that came to mind in response to a list of homographs and unambiguous filler words. The dominant meaning of the homographs had a probability range of .7-1 and a mean of .89 , and the subordinate meaning had a probability range of $0-.3$ and a mean of .11. The mean word length of the homographs was 4.93 (range $=3-8$ letters), and the mean SUBTLex word frequency was 48.02 occurrences per million (Brysbaert \& New, 2009).

Subjects read 5 practice sentences, followed by 120 experimental sentences and 80 nonexperimental filler sentences that were designed to mask the experiment's purpose. To provide sufficient power for the distributional analyses, the subjects were shown both the dominant and subordinate versions for all 60 of the sentence pairs. The order of trials was randomized, with the constraint that each homograph was read once in each half of the experiment, with 50 nonexperimental filler trials separating the two halves of the experiment. For each subject, half of the homographs appeared in the dominant sentence first, and half of the homographs appeared in the subordinate sentence first, and the order of the two versions of the sentence pairs was counterbalanced across subjects. All sentences were displayed on a single line, and the homographs never occupied the last two word positions of the sentence.

\section{Apparatus and procedure}

Eye movements were measured with an SR Research EyeLink 1000 system with high spatial resolution and a sampling rate of $1000 \mathrm{~Hz}$. Following calibration, gaze-position error was less than $0.5^{\circ}$. The sentences were displayed on a 21-in. ViewSonic monitor. All letters were lowercase (except when capitals were appropriate) and in a mono-spaced Courier font. The text was presented in black $\left(4.7 \mathrm{~cd} / \mathrm{m}^{2}\right)$ on a white background $\left(56 \mathrm{~cd} / \mathrm{m}^{2}\right)$. Subjects were seated $60 \mathrm{~cm}$ from the monitor, and 2.4 characters equaled approximately $1^{\circ}$ of visual angle. Subjects were instructed to read the sentences for comprehension. After reading each sentence, they pressed a button to end the trial and proceed to the next sentence. To ensure that subjects were reading for comprehension, about $20 \%$ of the sentences were followed by multiple-choice comprehension questions. The average accuracy rate was $97 \%$. 


\section{Results}

The main goal of the experiment was to examine the distributions of first-fixation durations on homographs as a function of the type of prior context (subordinate vs. dominant context conditions). In the Results section below, we begin by reporting analyses of mean fixation times, and we then report two types of distributional analyses: ex-Gaussian fitting (Staub et al., 2010) and a survival analysis technique (Reingold et al., 2012).

\section{Analysis of means}

Trials were excluded from the analyses described below due to track losses (less than $0.1 \%$ of all trials) and due to skipping of the target homograph (17.3\% of all trials). The following measures were used to compare fixation times on the homographs in the dominant context versus the subordinate context conditions: (1) first-fixation duration (i.e., the duration of the first forward fixation on the homograph, regardless of the number of subsequent fixations on the homograph), (2) gaze duration (i.e., the sum of all the consecutive first-pass fixations on the homograph, prior to a saccade to another word), (3) single-fixation duration (i.e., the first-fixation value for the subset of trials in which there was only one first-pass fixation on the homograph), (4) first of multiple first-pass fixations (i.e., the first-fixation duration for the subset of trials in which there was more than one first-pass fixation on the homograph), (5) go-past time (i.e., the sum of all fixations from the first fixation on the homograph up to and including the fixation prior to the reader's moving past the homograph to a later part of the sentence), (6) total time (i.e., the sum of all fixations on the homograph), (7) $n-1$ fixation (i.e., the duration of the fixation immediately before the first fixation on the homograph), (8) $n+1$ fixation (i.e., the duration of the fixation immediately after the final first-pass fixation on the homograph), (9) the probability of skipping (i.e., trials in which there was no first-pass fixation on the homograph, regardless of whether or not the homograph was fixated later in the trial), and (10) the probability of a single first-pass fixation. For all of these measures, planned comparisons by subjects $\left(t_{1}\right)$ and by items $\left(t_{2}\right)$ were performed across the two context conditions. Table 1 presents the means and standard errors of the different measures and the corresponding $t$ test results.

Table 1 Average fixation time measures (in milliseconds) and the probability (proportion) of skipping and single fixation by context condition

\begin{tabular}{|c|c|c|c|c|c|c|}
\hline \multirow[t]{3}{*}{ Measure } & \multicolumn{4}{|c|}{ Fixation time } & \multirow[t]{3}{*}{ Difference } & \multirow[t]{3}{*}{ Significance } \\
\hline & \multicolumn{2}{|c|}{ Subordinate Context } & \multicolumn{2}{|c|}{ Dominant Context } & & \\
\hline & $M$ & $S E$ & $M$ & $S E$ & & \\
\hline First-fixation (all trials) & 228 & 4.0 & 216 & 4.1 & 12 & $\begin{array}{l}t_{1}=5.88, p<.001 \\
t_{2}=4.16, p<.001\end{array}$ \\
\hline Gaze duration (all trials) & 259 & 6.5 & 241 & 5.8 & 18 & $\begin{array}{l}t_{1}=5.51, p<.001 \\
t_{2}=4.64, p<.001\end{array}$ \\
\hline Single fixation & 229 & 4.2 & 217 & 4.4 & 12 & $\begin{array}{l}t_{1}=6.48, p<.001 \\
t_{2}=3.93 p<.001\end{array}$ \\
\hline First fixation (multiple) & 230 & 6.5 & 212 & 6.5 & 18 & $\begin{array}{l}t_{1}=3.03, p<.01 \\
t_{2}=2.49, p<.05\end{array}$ \\
\hline Go-past time & 341 & 11.1 & 305 & 10.6 & 36 & $\begin{array}{l}t_{1}=5.83, p<.001 \\
t_{2}=4.51, p<.001\end{array}$ \\
\hline Total time & 371 & 13.1 & 316 & 11.5 & 55 & $\begin{array}{l}t_{1}=9.13, p<.001 \\
t_{2}=6.97, p<.001\end{array}$ \\
\hline$n-1$ fixation & 211 & 3.7 & 208 & 3.8 & 3 & $\begin{array}{l}t_{1}=1.91, p=.061 \\
t_{2}=1.51, p=.135\end{array}$ \\
\hline$n+1$ fixation & 235 & 3.9 & 229 & 4.3 & 6 & $\begin{array}{l}t_{1}=2.44, p<.05 \\
t_{2}=2.13, p<.05\end{array}$ \\
\hline Prob. of skipping & .17 & .01 & .18 & .01 & -.01 & $\begin{array}{l}t_{1}=1.53, p=.132 \\
t_{2}=1.54, p=.128\end{array}$ \\
\hline Prob. of single fixation & .71 & .01 & .71 & .01 & .00 & $\begin{array}{l}t_{1}<1 \\
t_{2}<1\end{array}$ \\
\hline
\end{tabular}

Note. For the $t$ tests shown above, $d f=59$. The means and standard errors shown are based on the by-subjects analyses 
In replication of the SBE (for a review, see Duffy et al., 2001), fixation times on the homographs were longer for the subordinate context condition relative to the dominant context condition. As is shown in Table 1, this effect was significant for first-fixation, gaze duration, single-fixation, first of multiple first-pass fixations, $n+1$ fixation, go-past time, and total time), and there were no significant effects for the remaining measures $(n-1$ fixation, probability of skipping, probability of a single first-pass fixation). Of particular interest to the present study's time course predictions, this pattern of results replicates previous demonstrations of significant lexical ambiguity effects on single-fixation and first-fixation durations (e.g., Kambe et al., 2001; Rayner et al., 2006; Sheridan et al., 2009) and extends this finding to the first of multiple first-pass fixations measure (for similar findings with a word frequency manipulation, see Reingold et al. 2012; Reingold, Yang, \& Rayner, 2010). Thus, the present demonstration of a significant SBE effect on early fixation time measures is consistent with the reordered access model's predictions concerning an early locus of contextual influences.

\section{Analyses of distributions}

Next, we report on the findings obtained from the analyses of the distribution of first-fixation durations by fitting fixation time data using the ex-Gaussian distribution, as well as by employing a survival analysis technique.

Fitting first-fixation duration with the ex-Gaussian distribution Figure 1a displays the distributions of firstfixation durations by condition (subordinate vs. dominant). To create this figure, we separately computed the proportion of first-fixation durations that fell within each successive 25$\mathrm{ms}$ bin over the range from 0 to $600 \mathrm{~ms}$ for each subject and each condition, and we then averaged these values across subjects. As can be seen from Fig. 1, distributions of firstfixation durations in reading tend to be approximately normal in shape, with some degree of rightward skew. Such distributions can be modeled using the ex-Gaussian distribution (e.g., Staub et al., 2010), which is the convolution of the Gaussian normal distribution and an exponential distribution. The shape of the ex-Gaussian distribution can be specified with three parameters: $\mu$ (the mean of the Gaussian component), $\sigma$ (the standard deviation of the Gaussian component), and $\tau$ (the mean and the standard deviation of the exponential component). Following Staub et al., we fitted the ex-Gaussian distribution to our first-fixation duration data using an algorithm known as quantile maximum likelihood estimation (QMPE; Cousineau, Brown, \& Heathcote, 2004; Heathcote, Brown, \& Mewhort, 2002). First-fixation duration data for each subject in each condition were fitted
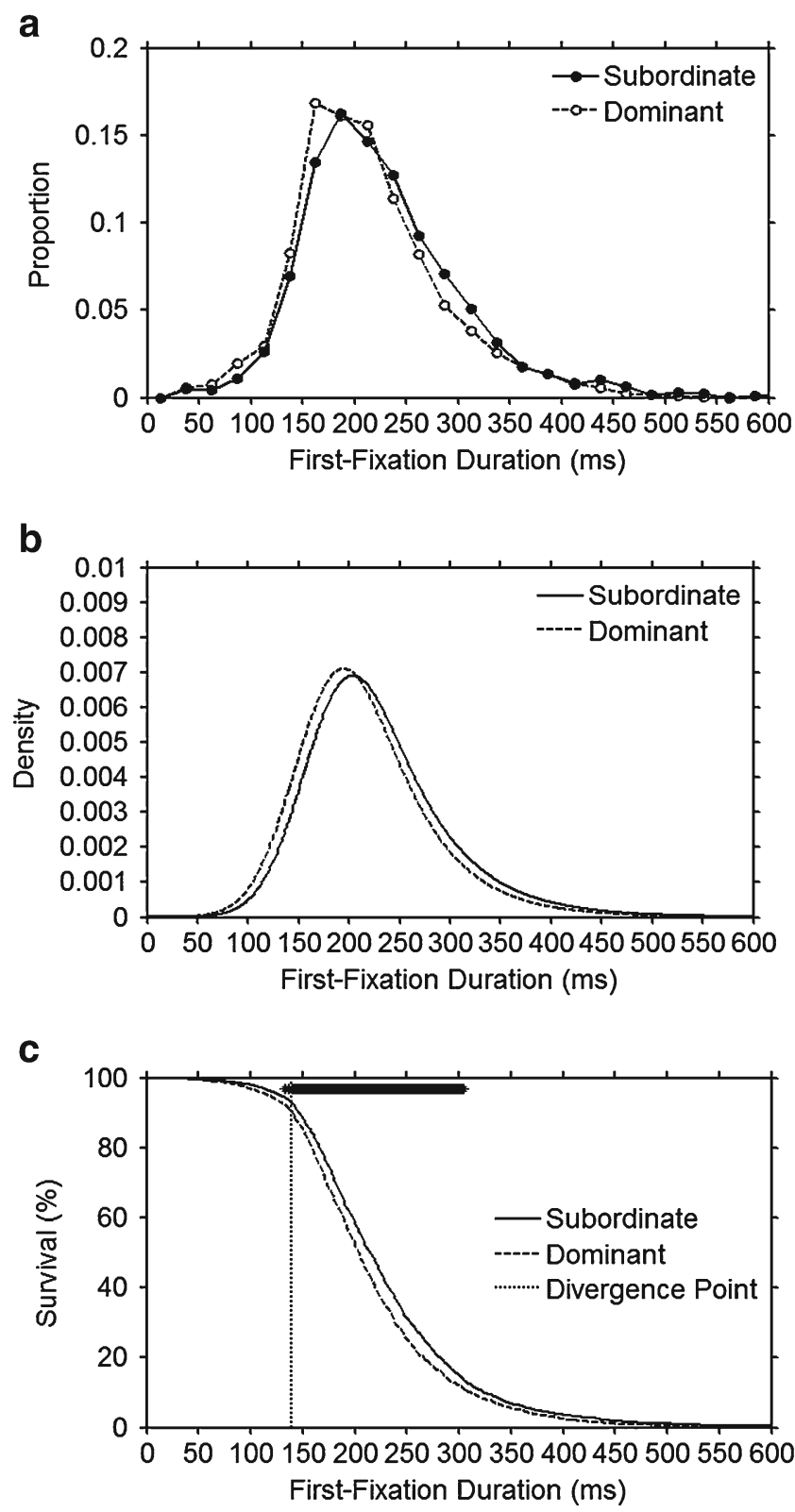

Fig. 1 Distributions of first-fixation duration on homographs in the subordinate and dominant conditions (a), ex-Gaussian density functions (b), and survival curves (c). The row of asterisks at the top of panel $\mathrm{c}$ indicates the time bins with a significant difference between the subordinate and dominant curves. See the text for further details

separately. There were an average of 50 usable observations per cell (see Table 2), and all fits successfully converged. Table 2 displays the mean number of usable observations per cell, the parameter estimates, and the magnitude and significance of the subordinate versus dominant context effects, and Fig. $1 \mathrm{~b}$ displays the density functions generated from the best-fitting ex-Gaussian parameters averaged across subjects.

As is shown in Table 2 and Fig. 1, the subordinate distribution was shifted to the right of the dominant distribution, resulting in a significant $\mu$ effect. There were no 
Table 2 Number of observation per cell and ex-Gaussian parameters by condition (with standard errors in parentheses)

\begin{tabular}{lllll}
\hline & $n$ & $\mathrm{Mu}(\mu)$ & Sigma $(\sigma)$ & Tau $(\tau)$ \\
\hline $\begin{array}{c}\text { Subordinate } \\
\text { sentence }\end{array}$ & $50(0.8)$ & $169(3.4)$ & $39(2.0)$ & $59(3.1)$ \\
$\begin{array}{c}\text { Dominant } \\
\text { sentence }\end{array}$ & $49(0.8)$ & $161(3.0)$ & $39(1.7)$ & $54(2.8)$ \\
$\begin{array}{c}\text { Difference } \\
\text { Significance }\end{array}$ & 1 & 8 & 0 & 5 \\
& $\begin{array}{c}t=1.55, \\
p=.127\end{array}$ & $\begin{array}{c}t=2.71, \\
p<.01\end{array}$ & $t<1$ & $\begin{array}{c}t=1.51, \\
p=.136\end{array}$ \\
\hline
\end{tabular}

Note. For the $t$ test results shown above, $d f=59$

significant differences for $\sigma$. There was a small numerical difference (subordinate $>$ dominant) for $\tau$, but this effect was not significant. This pattern of ex-Gaussian results supports the reordered access model's predictions concerning an early time course of contextual influences, because the presence of a shift in the distributions (i.e., the $\mu$ effect) indicates that the bulk of the fixations were influenced by the contextual manipulation, regardless of their duration. More generally, the present study's $\mu$ effect is consistent with similar findings that were produced by manipulations of other lexical variables, including word frequency (Reingold et al., 2012; Staub et al., 2010) and predictability or contextual constraints (Staub, 2011).

Survival analysis We computed survival curves for firstfixation durations in the subordinate and dominant context conditions, using the same procedure as that in Reingold et al. (2012). Specifically, for each 1-ms time bin $t$ ( $t$ was varied from 0 to $600 \mathrm{~ms}$ ), the percentage of first fixations with a duration greater than $t$ constituted the percent survival at time $t$. The survival curve was computed separately for each condition and for each subject and then was averaged across subjects. As is shown in Fig. 1c, the subordinate and dominant survival curves appear to diverge. Importantly, this divergence point corresponds by definition to the shortest first-fixation duration value at which the contextual manipulation had a significant impact. To estimate the divergence point, we employed a bootstrap resampling procedure (Efron \& Tibshirani, 1994). The procedure that we used is outlined in detail by Reingold et al. (2012). On each iteration of this procedure, the set of observations (first-fixation durations) for each subject in each condition was randomly resampled with replacement. For each iteration of the bootstrap procedure, individual subject's survival curves were then computed and averaged. Next, the value for each 1-ms bin in the dominant survival curve was subtracted from the corresponding value in the subordinate survival curve. This procedure was repeated 10,000 times, and the obtained differences for each bin were then sorted in order of magnitude. The range between the 5 th and the 9,995th value was then defined as the confidence interval of the difference for each bin (given the multiple comparisons we performed, we used this conservative confidence interval in order to protect against making a Type I error). To compute the divergence point between the subordinate and dominant survival curves, we identified the time bins for which the subordinate survival rate was significantly greater than the dominant survival rate (i.e., for which the lower bound of the confidence interval of the difference between the subordinate and dominant curves was greater than zero). The divergence point was then defined as the earliest significant difference point that was part of a run of five consecutive significant difference points (significant differences between the subordinate and dominant curves are shown in Fig. $1 \mathrm{c}$ as a row asterisks above the survival curves).

As can be seen in Fig. 1c, the dominant and subordinate survival curves significantly diverged at a duration of $139 \mathrm{~ms}$, which is similar to the 145 -ms divergence point that was previously obtained for the word frequency variable under normal reading conditions (see Reingold et al., 2012). Furthermore, the divergence point defines the percentage of first fixations with durations that were too short to exhibit an influence of lexical ambiguity. In the present study, only approximately $8 \%$ of first fixations had durations that were shorter than the divergence point, which is once again similar to the prior word frequency findings (Reingold et al., 2012). Given that word frequency effects are often used as an empirical marker of lexical processing (see Rayner, 1998, 2009), the fact that the present study's temporal estimates are equally as rapid as the word frequency estimates provides strong evidence for an early locus of contextual influences during lexical ambiguity resolution. Taken together, the ex-Gaussian and survival analysis results are consistent with the reordered access model's assumption that contextual influences can impact lexical access and are difficult to reconcile with the integration model's assumption that contextual influences are postlexical.

\section{Discussion}

The present findings provide strong support for an early locus of contextual influences during lexical ambiguity resolution. Specifically, to provide fine-grained time course information, the present study examined both mean and distributional analyses of fixation times on homographs in a dominant-instantiating versus a subordinate-instantiating context condition. This contextual manipulation replicated the SBE (for a review, see Duffy et al., 2001) by producing longer mean fixation times in the subordinate than in the dominant condition across a variety of early fixation duration measures, including first-fixation, single-fixation, and first in multiple first-pass fixations. Furthermore, the distributional analyses supported an early time course of contextual 
influences. In particular, the ex-Guassian analysis revealed that the subordinate distribution was shifted to the right of the dominant distribution (i.e., a significant $\mu$ effect), and the survival analysis revealed that an effect of the contextual manipulation on first-fixation duration was evident as early as $139 \mathrm{~ms}$ from the start of the fixation.

Consistent with the present findings, ERP work has demonstrated rapid contextual influences on the $\mathrm{N} 1$ component (132-192 ms post-stimulus-onset) during lexical ambiguity resolution (Sereno et al., 2003). Moreover, several previous lexical ambiguity studies have shown contextual effects on the duration of the first fixation on the homograph (e.g., Kambe et al., 2001; Rayner et al., 2006; Sheridan et al., 2009), and the SBE has been demonstrated using a single adjective immediately preceding the homograph (e.g., table), such that fixation times on the homograph were longer following a subordinate-instantiating (e.g., statistical table), relative to a dominant-instantiating (e.g., kitchen table), prior context (Rayner et al., 2006). Consistent with these empirical findings, a simulation by Reichle et al. (2007) incorporated the assumption that contextual influences during lexical ambiguity resolution are rapid enough to influence the earliest stage of lexical processing (i.e., the $\mathrm{L}_{1}$ stage in the $\mathrm{E}-\mathrm{Z}$ Reader model; Reichle, Pollatsek, Fisher, \& Rayner, 1998; Reichle, Warren, \& McConnell, 2009)

Of relevance to contemporary theories of lexical ambiguity resolution, rapid contextual influences are consistent with the reordered access model's assumption that context can influence lexical access and are inconsistent with the integration model's assumption that context does not play a role until the postlexical integration stage. More specifically, to explain the SBE, the reordered access model (Duffy et al., 1988) assumes that a subordinate-instantiating context can influence lexical access by speeding up the access of the subordinate meaning such that it can compete with the dominant meaning. In marked contrast, the integration model (Rayner \& Frazier, 1989) contends that the SBE stems from processing difficulty during the postlexical integration stage. According to the integration model, the SBE occurs because readers fail to integrate the dominant meaning and must then wait for the subordinate meaning to become available in order for integration to proceed. To explain why the SBE emerges as early as the first fixation on the homograph, the integration model could contend that such effects were driven by a small subset of trials with fixation durations that were long enough to enable integration processes to play a role. However, this explanation is inconsistent with the present study's distributional analyses, because both the ex-Gaussian and the survival analyses demonstrated contextual influences on the vast majority of fixations. Consequently, the present study's results add to a growing body of evidence that supports the reordered access model (Dopkins et al., 1992; Duffy et al., 2001; Reichle et al., 2007; Sereno, 1995; Sereno et al., 2003; Sheridan et al., 2009).
More generally, lexical ambiguity research has historically played a prominent role in the larger debate over whether lexical access is impacted by contextual influences (i.e., the interactive perspective; McClelland \& Rumelhart, 1981; Morton, 1969) versus impervious to contextual influences (i.e., the modular perspective; Fodor, 1983; Forster, 1979). Similar to previous work on this topic (e.g., Sereno et al., 2003), the present study's results favor the interactive perspective. However, the present study's results do not adjudicate between strong interactive accounts that assume that context can lead to selective access of the contextually relevant meaning (e.g., Kellas \& Vu, 1999; Vu \& Kellas, 1999) and hybrid accounts, such as the reordered access model (Duffy et al., 1988), which, instead, assumes that context can "reorder" lexical access by speeding the access of the contextually relevant meaning without producing selective access. Rather, the present study's results support the interactive perspective more generally by demonstrating that the subordinate versus dominant contextual manipulation can produce a fast-acting influence on fixation durations.

In addition, the present findings are relevant to ongoing debates over competing models of eye movement control during reading. In this controversy, one class of models assumes that fixation times are driven primarily by visual/ oculomotor factors and that lexical variables can impact only a small subset of long fixations, whereas a competing class of models assumes that lexical variables can have a fast-acting influence on the majority of fixation times during reading (see Rayner, 1998, 2009, for reviews). In support of the latter class of models, the present study's results coincide with previous findings that lexical variables in reading are fast-acting. Such findings include the dramatic demonstration that word frequency effects still occur even when the text disappears $60 \mathrm{~ms}$ into the fixation (Rayner, Liversedge, White, \& Vergilino-Perez, 2003) and the finding that a wide range of lexical variables can impact first-fixation duration, including lexical ambiguity (see Duffy et al., 2001, for a review), word frequency (see White, 2008, for a review), predictability or contextual constraint (e.g., Ehrlich \& Rayner, 1981; Staub, 2011), and age of acquisition (e.g., Juhasz \& Rayner, 2006). More recently, ex-Gaussian analyses have shown that lexical variables can produce shifts in distributions of fixation duration, which indicates that they are impacting the majority of fixations, regardless of duration. For example, the present study's lexical ambiguity manipulation produced a shift in first-fixation durations, and prior work has shown a similar shift effect for word frequency (Reingold et al., 2012; Staub et al., 2010) and predictability (Staub, 2011). Finally, survival curve analyses have produced rapid temporal estimates of the earliest impact of lexical variables on fixation times, both in the present study and in prior work concerning word frequency (Reingold et al., 2012). Taken together, these findings support models of eye movement control that 
postulate fast-acting direct lexical control of fixation times during reading (see Rayner, 1998, 2009, for reviews).

In sum, the present study provides strong support for a rapid time course of contextual influences during lexical ambiguity resolution. This pattern of results fits well with the time course predictions of the reordered access model and is inconsistent with the integration model. Moreover, of relevance to models of eye movement control, the present study's results provide convergent evidence for an early time course of lexical influences during reading. Finally, the present study demonstrates that distributional analysis techniques can provide a useful method for studying the time course of lexical ambiguity effects. Future research could further employ these techniques to examine the time course of other types of ambiguity effects (e.g., syntactic ambiguity, garden path sentences, etc.).

\section{References}

Binder, K. S. (2003). Sentential and discourse topic effects on lexical ambiguity processing: An eye movement examination. Memory \& Cognition, 31, 690-702.

Binder, K. S., \& Rayner, K. (1999). Does contextual strength modulate the subordinate bias effect? A reply to Kellas and Vu. Psychonomic Bulletin \& Review, 6, 518-522. doi:10.3758/BF03210843

Brysbaert, M., \& New, B. (2009). Moving beyond Kučera and Francis: A critical evaluation of current word frequency norms and the introduction of a new and improved word frequency measure for American English. Behavior Research Methods, 41, 977-990. doi:10.3758/BRM.41.4.977

Cousineau, D., Brown, S., \& Heathcote, A. (2004). Fitting distributions using maximum likelihood: Methods and packages. Behavior Research Methods, Instruments, \& Computers, 36, 742-756.

Dopkins, S., Morris, R. K., \& Rayner, K. (1992). Lexical ambiguity and eye fixations in reading: A test of competing models of lexical ambiguity resolution. Journal of Memory and Language, 31, 461476. Elsevier.

Duffy, S. A., Kambe, G., \& Rayner, K. (2001). The effect of prior disambiguating context on the commehension of ambiguous words: Evidence from eye movements. In D. S. Gorfein (Ed.), On the consequences of meaning selection: Perspectives on resolving lexical ambiguity (pp. 27-43). Washington, DC: American Psychological Association.

Duffy, S. A., Morris, R. K., \& Rayner, K. (1988). Lexical ambiguity and fixation times in reading. Journal of Memory and Language, 27, 429-446. doi:10.1016/0749-596X(88)90066-6

Efron, B., \& Tibshirani, R. J. (1994). An introduction to the bootstrap. Boca Raton, FL: Chapman \& Hall.

Ehrlich, S. F., \& Rayner, K. (1981). Contextual effects on word perception and eye movements during reading. Journal of Verbal Learning and Verbal Behavior, 20, 641-655.

Feng, G., Miller, K., Shu, H., \& Zhang, H. (2001). Rowed to recovery: The use of phonological and orthographic information in reading Chinese and English. Journal of Experimental Psychology: Learning, Memory, and Cognition, 27, 1079-1100.

Fodor, J. A. (1983). The modularity of mind. Cambridge: MIT Press.

Folk, J. R., \& Morris, R. K. (2003). Effects of syntactic category assignment on lexical ambiguity resolution in reading: An eye movement analysis. Memory \& Cognition, 31, 87-99.

Forster, K. I. (1979). Levels of processing and the structure of the language processor. In W. E. Cooper \& E. C. T. Walker (Eds.), Sentence processing: Psycholinguistic studies presented to Merrill Garrett (pp. 27-85). Hillsdale: Erlbaum.
Glaholt, M. G., \& Reingold, E. M. (in press). Direct control of fixation times in scene viewing: Evidence from analysis of the distribution of first fixation duration. Visual Cognition. doi:10.1080/ 13506285.2012.666295

Heathcote, A., Brown, S., \& Mewhort, D. J. K. (2002). Quantile maximum likelihood estimation of response time distributions. Psychonomic Bulletin \& Review, 9, 394-401.

Hogaboam, T. W., \& Perfetti, C. A. (1975). Lexical ambiguity and sentence comprehension. Journal of Verbal Learning \& Verbal Behavior, 14(3), 265-274.

Juhasz, B. J., \& Rayner, K. (2006). The role of age of acquisition and word frequency in reading: Evidence from eye fixation durations. Visual Cognition, 13, 846-863.

Kambe, G., Rayner, K., \& Duffy, S. A. (2001). Global context effects on processing lexically ambiguous words: Evidence from eye fixations. Memory \& Cognition, 29, 363-372.

Kellas, G., \& Vu, H. (1999). Strength of context does modulate the subordinate bias effect: A reply to Binder and Rayner. Psychonomic Bulletin \& Review, 6, 511-517.

Lucas, M. (1999). Context effects in lexical access: A meta-analysis. Memory \& Cognition, 27, 385-398.

McClelland, J. L., \& Rumelhart, D. E. (1981). An interactive activation model of context effects in letter perception: Part 1. An account of basic findings. Psychological Review, 88, 375-407.

Morton, J. (1969). Interaction of information in word recognition. Psychological Review, 76, 165-178.

Pacht, J. M., \& Rayner, K. (1993). The processing of homophonic homographs during reading: evidence from eye movement studies. Journal of Psycholinguistic Research, 22, 251-271.

Ratcliff, R. (1979). Group reaction time distributions and an analysis of distribution statistics. Psychological Bulletin, 86, 446-461.

Rayner, K. (1998). Eye movements in reading and information processing: 20 years of research. Psychological Bulletin, 124, 372422.

Rayner, K. (2009). Eye movements in reading: Models and data. Journal of Eye Movement Research, 2, 1-10.

Rayner, K., Binder, K. S., \& Duffy, S. A. (1999). Contextual strength and the subordinate bias effect: Comment on Martin, Vu, Kellas, and Metcalf. Quarterly Journal of Experimental Psychology, 52A, 841-852. doi:10.1080/713755868

Rayner, K., Cook, A. E., Juhasz, B. J., \& Frazier, L. (2006). Immediate disambiguation of lexically ambiguous words during reading: Evidence from eye movements. British Journal of Psychology, 97, 467-482.

Rayner, K., \& Duffy, S. A. (1986). Lexical complexity and fixation times in reading: Effects of word frequency, verb complexity, and lexical ambiguity. Memory \& Cognition, 14, 191-201.

Rayner, K., \& Frazier, L. (1989). Selection mechanisms in reading lexically ambiguous words. Journal of Experimental Psychology: Learning, Memory, and Cognition, 15, 779-790.

Rayner, K., Liversedge, S. P., White, S. J., \& Vergilino-Perez, D. (2003). Reading disappearing text: Cognitive control of eye movements. Psychological science, 14, 385-388.

Rayner, K., Pacht, J. M., \& Duffy, S. A. (1994). Effects of prior encounter and global discourse bias on the processing of lexically ambiguous words: Evidence from eye fixations. Journal of Memory and Language, 33, 527-544.

Reichle, E. D., Pollatsek, A., Fisher, D. L., \& Rayner, K. (1998). Toward a model of eye movement control in reading. Psychological Review, 105, 125-157.

Reichle, E. D., Pollatsek, A., \& Rayner, K. (2007). Modeling the effects of lexical ambiguity on eye movements during reading. In R. P. G. van Gompel, M. H. Fischer, W. S. Murray, \& R. L. Hill (Eds.), Eye movements: A window on mind and brain (pp. 271-292). Oxford: Elsevier.

Reichle, E. D., Warren, T., \& McConnell, K. (2009). Using E-Z Reader to model the effects of higher level language processing on eye 
movements during reading. Psychonomic Bulletin \& Review, 16, 1-21. doi:10.3758/PBR.16.1.1

Reingold, E. M., Reichle, E. D., Glaholt, M. G., \& Sheridan, H. (2012). Direct lexical control of eye movements in reading: Evidence from a survival analysis of fixation durations. Cognitive Psychology, 65, 177-206. doi: 10.1016/j.cogpsych.2012.03.001

Reingold, E. M., \& Sheridan, H. (2011). Eye movements and visual expertise in chess and medicine. In S. P. Liversedge, I. D. Gilchrist, \& S. Everling (Eds.), Oxford handbook on eye movements (pp. 528-550). Oxford: Oxford University Press.

Reingold, E. M., Yang, J., \& Rayner, K. (2010). The time course of word frequency and case alternation effects on fixation times in reading: Evidence for lexical control of eye movements. Journal of Experimental Psychology: Human Perception and Performance, 36, 1677-1683. doi:10.1037/a0019959

Sereno, S. C. (1995). Resolution of lexical ambiguity: Evidence from an eye movement priming paradigm. Journal of Experimental Psychology: Learning, Memory, and Cognition, 21, 582-595.

Sereno, S. C., Brewer, C. C., \& O’Donnell, P. J. (2003). Context effects in word recognition: Evidence for early interactive processing. Psychological Science, 14, 328-333.

Sereno, S. C., O’Donnell, P. J., \& Rayner, K. (2006). Eye movements and lexical ambiguity resolution: Investigating the subordinate-bias effect. Journal of Experimental Psychology: Human Perception and Performance, 32, 335-350. doi:10.1037/0096-1523.32.2.335

Sereno, S. C., Pacht, J. M., \& Rayner, K. (1992). The effect of meaning frequency on processing lexically ambiguous words: Evidence from eye fixations. Psychological Science, 3, 296-300. doi:10.1111/j.1467-9280.1992.tb00676.x

Sereno, S. C., \& Rayner, K. (2003). Measuring word recognition in reading: Eye movements and event-related potentials. Trends in Cognitive Sciences, 7, 489-493. doi:10.1016/j.tics.2003.09.010

Sheridan, H., Reingold, E. M., \& Daneman, M. (2009). Using puns to study contextual influences on lexical ambiguity resolution: Evidence from eye movements. Psychonomic Bulletin \& Review, 16, 875-881. doi:10.3758/PBR.16.5.875
Sheridan, H., \& Reingold, E. M. (in press). The time course of predictability effects in reading: Evidence from a survival analysis of fixation durations. Visual Cognition.

Staub, A. (2011). The effect of lexical predictability on distributions of eye fixation durations. Psychonomic Bulletin \& Review, 18, 371-376. doi:10.3758/s13423-010-0046-9

Staub, A., White, S. J., Drieghe, D., Hollway, E. C., \& Rayner, K. (2010). Distributional effects of word frequency on eye fixation durations. Journal of Experimental Psychology: Human Perception and Performance, 36, 1280-1293. doi:10.1037/a0016896

Swinney, D. A. (1979). Lexical access during sentence comprehension: (Re)consideration of context effects. Journal of Verbal Learning and Verbal Behavior, 18, 645-659. doi:10.1016/S0022-5371(79)90355-4

Vu, H., \& Kellas, G. (1999). Contextual strength modulates the subordinate bias effect: Reply to Rayner, Binder, and Duffy. Quarterly Journal of Experimental Psychology, 52A, 853-855. doi:10.1080/713755863

White, S. J. (2008). Eye movement control during reading: Effects of word frequency and orthographic familiarity. Journal of Experimental Psychology: Human Perception and Performance, 34, 205-223. doi:10.1037/0096-1523.34.1.205

White, S. J., \& Staub, A. (in press). The distribution of fixation durations during reading: Effects of stimulus quality. Journal of Experimental Psychology: Human Perception and Performance. doi:10.1037/a0025338

White, S. J., Staub, A., Drieghe, D., \& Liversedge, S. P. (2011, August). Word frequency effects during reading: Binocular fixations and the distribution of fixation durations. In 16th European Conference on Eye Movements (ECEM). Marseille, France.

White, S. J., Warren, T., Staub, A., \& Reichle, E. D. (2011, August). The distribution of fixation durations during reading: Effects of stimulus quality and sentence wrap-up. In 16th European Conference on Eye Movements (ECEM). Marseille, France.

Wiley, J., \& Rayner, K. (2000). Effects of titles on the processing of text and lexically ambiguous words: Evidence from eye movements. Memory \& Cognition, 28, 1011-1021. 